\title{
RELIABILITY OF ELECTRONICS
}

\author{
Larry E. Wickstrom
}

Thesis Prepared for the Degree of

MASTER OF SCIENCE

\section{UNIVERSITY OF NORTH TEXAS}

December 2014

APPROVED:

Hyoung Soo Kim, Major Professor Hualiang Zhang, Committee Member Gayatri Mehta, Committee Member Shengli Fu, Committee Member and Chair of the Department of Electrical Engineering

Kuruvilla John, Associate Dean of the College of Engineering

Costas Tsatsoulis, Dean of the College of Engineering

Mark Wardell, Dean of the Toulouse Graduate School 
Wickstrom, Larry E. Reliability of Electronics. Master of Science (Electrical Engineering), December 2014, 51 pp., 16 figures, bibliography, 10 titles.

The purpose of this research is not to research new technology but how to improve existing technology and understand how the manufacturing process works. Reliability engineering falls under the category of quality control and uses predictions through statistical measurements and life testing to figure out if a specific manufacturing technique will meet customer satisfaction. The research also answers choice of materials and choice of manufacturing process to provide a device that will not only meet but exceed customer demand. Reliability engineering is one of the final testing phases of any new product development or redesign. 
Copyright 2014

by

Larry E. Wickstrom 


\section{ACKNOWLEDGEMENTS}

Over the past three years I have enjoyed working with Prof. Hyoung Soo Kim as my advisor and I would like to thank him for all his guidance and support over the course of my Graduate Studies at the University of North Texas. I would also like to thank Prof's Shengli Fu, Hualiang Zhang, and Gayatri Mehta for taking part as members of my thesis committee and for having me in their classes. 


\section{TABLE OF CONTENTS}

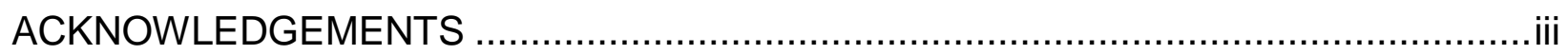

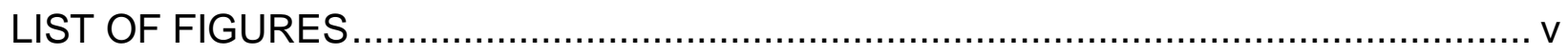

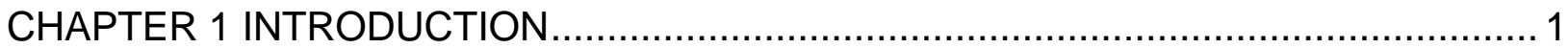

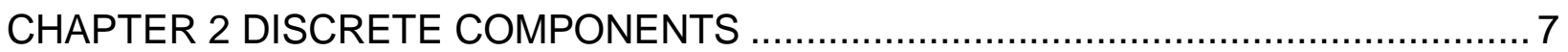

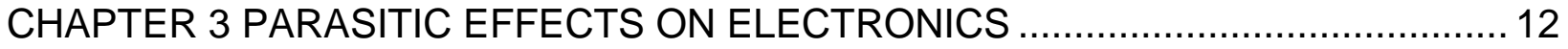

CHAPTER 4 COMPONENT RELIABILITY ....................................................... 22

CHAPTER 5 MATERIALS USED IN SEMICONDUCTORS ................................. 38

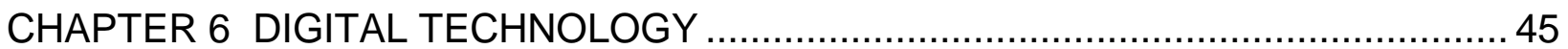

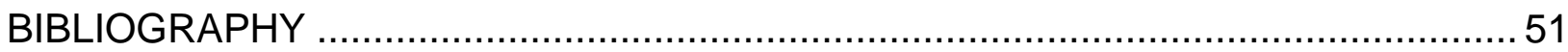




\section{LIST OF FIGURES}

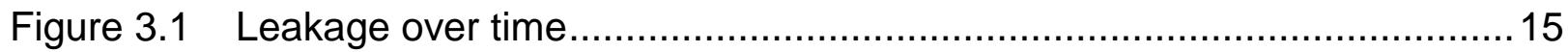

Figure 3.2 Leakage current over extended periods ............................................15

Figure 3.3 Leakage current over time and temperature ….................................16

Figure 3.4 Plexiglas after breakdown voltage limit was applied and reached............17

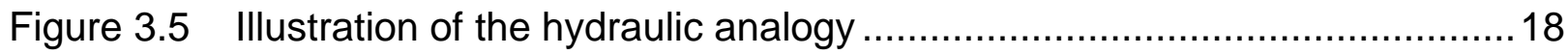

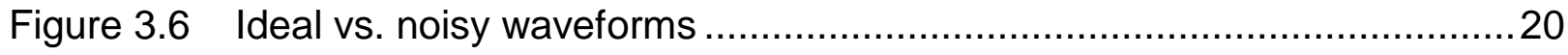

Figure 3.7 High pass and low pass circuit examples........................................21

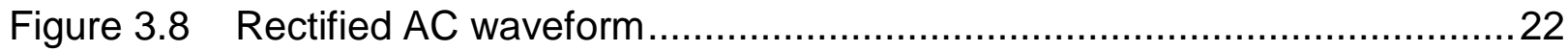

Figure 4.1 Capacitor types and typical values ................................................28

Figure 4.2 Simple parallel plate capacitor design .............................................29

Figure 4.3 Multi-layer ceramic chip capacitor cross section .................................30

Figure 4.4 Defects of chip capacitors due to high temp or high wattage ..................30

Figure 4.5 Internal components of an electrolytic capacitor ...................................34

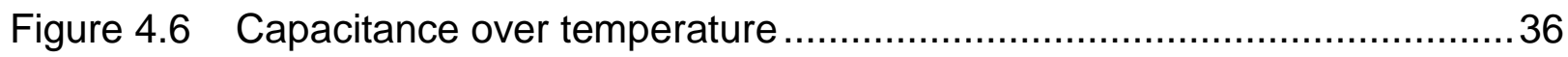

Figure $4.7 \quad$ Examples of failed electrolytic capacitors...............................................

Figure $4.8 \quad$ Electrolytic capacitor lifespan over temperature .....................................38 


\section{CHAPTER 1}

\section{INTRODUCTION}

Why do electronic devices behave differently when running at full capacity or in abnormal environmental conditions?

The purpose of this study is to look at common knowledge about electronics and quantify it with data and in detailed explanations. This area of research mostly covers reliability or quality assurance (QA) engineering topics. I chose these topics because this has been an important part of my jobs in industry over the past decade. Another very important topic covered is the parts designed for specific applications that require heavy stress, load, or extreme environmental conditions.

So far, silicon has been the main base material for semiconductor devices and has been an industry standard since the invention of the transistor as well as other solid state electronics. This is because silicon is a pure element and does not present the flaws and issues that compound base materials like gallium arsenide (GaAs) do. Other materials and elements can be introduced, or doped, into silicon without affecting its material or crystal lattice structure. Silicon with these added materials presents many problems that need to be addressed in order to prevent usage failures.

The upcoming issue with silicon is that its theoretical switching distance limit is about to be reached. Physicists have claimed that $10 \mathrm{~nm}$ is the theoretical limit that a charge can be transferred from point to point, making $5 \mathrm{~nm}$ lithography on silicon the lowest known possible transistor line size. At this point, $22 \mathrm{~nm}$ technology, and soon $14 \mathrm{~nm}$, will be readily available to the public. This is why other materials, like GaAs, are being researched with faster switching potential and smaller transistor sizes. Not only 
are different materials being looked into but also new switching methods and transistor shapes on new materials and existing silicon are also being researched because the $5 \mathrm{~nm}$ limit is well known and new solutions are always being found.

Another piece of common knowledge is Ohm's Law: I = V/R, everyone in the engineering field knows of this and its mathematical properties. However there is a major difference between an ideal mathematical situation and a real world situation. Some devices focus on current values and some on voltage values but the resistance is the constant that is dialed in to affect one or the other (Power (W) is an effect of $I^{*} V$ and is included in this) in its operation. However resistance will change under different conditions affecting the other components of the desired result.

A semiconductor is what the name says, it is neither a full conductor or insulator. It performs according to the materials introduced and the charges applied. Every material, even full metal conductors like copper, have resistance. These materials are affected by heat, stress, and wear. As it is well known when materials heat up they expand and the reverse for cooling and when materials expand the resistance also goes up. Now when you look at a semiconductor feature say from $25 \mathrm{C}$ to $85 \mathrm{C}$ in temp it does not expand that much maybe a few nanometers or microns depending on the feature size, right? Now when you have a chip that has millions or billions or transistors all expanding and contracting based on current flow or environment then it becomes a big deal.

Switching speeds and amount of current are one of the major sources for heat in chips. This added with heat buildup from processing and outside environmental heat or cold can add to complications in design. This is where a specific design or 
recommended usage rating comes from. Most consumer grade products are rated for $0 \mathrm{C}$ to $85 \mathrm{C}$ in that range but other uses will require either a different or wider range. This is where the choice of materials, design structure, and design parameters play a large role.

The purpose of this research is to quantify common knowledge. As stated before most consumer grade products have a specific operating temperature and humidity rating. The reason the range is limited, compared to others, for consumer use is for reasons of cost and device yield in fabrication. Commercial, industrial, or automotive requirements are much stricter. In this the cost per device goes up. The same chip rated for consumer grade can cost much more per chip with wider requirement needs. In the case for automotive, safety and reliability is the largest concern and special precautions must be taken to insure higher quality and operating range.

As for cost, I am sure the readers have heard about microchips ranging in the five to seven figure cost range and think to themselves: "How is this possible when I can pick up an Intel CPU for $\$ 100$ at Fry's electronics?" The reason is that the same chip is required to have either: a much lower margin of error during test, a customer specific addition or application, or require an operating range so extreme that the average consumer cannot comprehend it. Let's take a chip that is needed for satellite, in outer space the temperature can range from absolute zero (-273C) to much higher temps along with unblocked radiation. It is very difficult to test these conditions and when tested, only a small percentage of chips will survive probe, final, or field testing.

All the added outside effects can alter the operation of any chip more so in analog devices than others. Analog technology requires precision for precision values is 
how analog processing works. Analog is a continuous signal sent through any medium most by wire or by air. The signal needs to be read properly which is where the noise and interference factors come in. Before digital technology came along analog processing required a lot of filtering for error correction without the aid of digital processing. This was usually in the form of a lot of resistors, capacitors, inductors, and other discrete devices. Hence the reason why high tech was so expensive pre-dating digital (take inflation into consideration).

To obtain perfect point to point analog transmission with no errors became more expensive the further the distance was. This is why most consumer products (I'm thinking 1980s and before here) allowed a certain amount of error to occur to keep costs down. For those who remember how important TV signal reception was before cable TV came in will know how imperfect an over the air transmission was, especially for those who lived far enough away from the station antenna. The static showed the error I am speaking of, anything from bad weather to white noise could interfere with the point to point transmission over distance. This on top of the fact that most consumer grade parts did not last nearly as long as they did now due to heat, usage, and high energy requirements lead to the desire for digital technology.

Digital technology became main stream in the consumer market in the 1990 s. Even though digital technology was around much longer and beginning to creep into the market earlier, the mid1990s is when digital technology really took off. Digital is what the word represents: the use of digits for calculations. Digital technology makes use of discrete values, mostly in the form of binary numbers representing a 1 or 0 . In semiconductors the 1 and 0 values are represented by a set voltage, usually ground is 
the 0 and the supply voltage is the 1 . Digital offers a solution compared to analog in terms of margin of error and error correcting. A voltage range can be set to represent a high or low value eliminating the need for a precision value. Calculating binary values helps make error correcting much easier and cheaper since the need for discrete components required for analog filtering and processing is greatly reduced.

Digital processing has improved greatly over the last decade. Digital filtering and processing are two techniques have improved. The improvements come at a cost, higher clock speeds and constant re-designs are required to meet the demand. TDP or thermal design power has been an issue in processing for some time. Since lithography sizes are decreasing more transistors are capable of fitting in modern CPUs this means more switching operations per second are taking place hence higher power consumption. One benefit of smaller transistor size is lower power consumption per transistor but with the multiplied count of transistors the natural lower power consumption is not enough. Voltage settings have also decreased to compensate for the overall wattage within but this required major changes in transistor design to be used at lower levels. At one point in time 5 volts was the standard for digital switching; now we are as low as $1-1.5 \mathrm{~V}$. Smaller transistor sizes and lower power usage also introduce more design complications but this is discussed later on. To sum up the TDP issue a CPU that now uses $1-1.5 \mathrm{~V}$ consumes a total of $70-150$ watts on standard desktop applications to give an idea on how much goes on in modern CPUs.

While digital technology offers a more accurate and cost effective solution in some cases it is not a replacement for analog technology. With wireless technology becoming more widespread the demand for analog technology surged back. Digital DC 
voltage levels cannot be transmitted over the air, it requires a discrete or continuous wave to do it which is why analog is required. However it is possible to send a specific analog signal with a digital representation over the air. This is one example of why both technologies are needed and how they are used together. There are also other ways multiple technologies can help complement one another. There are more examples in the sections that follow. 


\section{CHAPTER 2}

\section{DISCRETE COMPONENTS}

A major part of analog and digital technology is the use of discrete components. In design we often think of the mathematical representation of these components and the effects that they will produce. In practice other design methods are added to insure device reliability and component lifespan.

Resistance is always present in any discrete component or material, though the resistance is dependent on the operation of the component and materials used. The basic carbon resistor and copper wire are good examples to start out with because they are used in just about every electronic device and the resistance is relatively simple to understand and it is not affected by as many factors. Even though the readers are likely to know this, wires are used to conduct electrical current and resistors are used to restrict the flow of electrical current.

Temperature dependence is one of the factors that affects the value of resistance and can affect device performance depending on the accuracy required. In most clean rooms, server rooms, laboratories, etc the environment is temperature controlled for this very reason. Room temperature is usually defined as 20 or 25 degrees Celsius which is 68 to 77 Fahrenheit. Most controlled environments I have been in use 70F or $20 \mathrm{C}$ as room temperature since it is a common standard for calculations.

Every material used has a resistance and temperature coefficient, in wire copper is commonly used and in resistors carbon is used as stated before. Assuming we have materials with no impurities the electrical resistance of a wire is: 
$\mathrm{R}=\rho \mathrm{L} / \mathrm{A}$

$R=$ Resistance $(\Omega), \rho=$ Resistivity Coefficient $(\Omega m), L=$ Length of Wire $(m), A=$ Cross section area of wire $\left(\mathrm{m}^{2}\right)$

Take 10 gauge wire which is the largest that will be commonly seen the cross section of the area is $2.59 \mathrm{~mm}\left(5.26 \mathrm{~mm}^{2}\right), 1$ meter of wire, and a coefficient for copper is $1.724 * 10^{-8}$.

$$
R=\left(1.724 * 10^{-8} * 1 \mathrm{~m}\right) /\left(5.26 \mathrm{~mm}^{2} * 10^{-6} \mathrm{~m}^{2} / \mathrm{mm}^{2}\right)=3.277^{*} 10^{-3} \Omega \text { over } 1 \mathrm{~m} \text { of } 10
$$
gauge wire.

For $1 \mathrm{~m}$ of 20 gauge which is commonly used in electronics (most cases smaller):

$$
R=\left(1.724^{*} 10^{-8} * 1 \mathrm{~m}\right) /\left(0.518 \mathrm{~mm}^{2} * 10^{-6} \mathrm{~m}^{2} / \mathrm{mm}^{2}\right)=33.281 * 10-3 \Omega \text { over } 1 \mathrm{~m} \text { of } 20
$$
gauge wire.

Even though the resistance is not high over copper wire this shows that 20 gauge has $10 x$ the resistance of 10 gauge wire over the same distance. This directly affects the maximum ampere rating for wire for power transmission, 10 gauge has a $15 \mathrm{~A}$ rating and 20 gauge has a $1.5 \mathrm{~A}$ rating. However there is a trade off in the terms of maximum frequency where 10 gauge can transmit $2600 \mathrm{~Hz}$ and 20 gauge can transmit $26 \mathrm{kHz}$ which is why smaller gauge wire is desired in electronics but distance must always be accounted for. (Source: American Wire Gauge Standard.)

Over temperature these ratings change. It is not only important to know for reasons of environment but also when wattage is transmitted over any discrete component the temperature of it will go up. Since carbon resistors are set by the manufacturer I will not go into specifics of the dimensions of the carbon film to set resistance. Copper wire will also change in resistance over temperature, this is the 
exact reason why max ampere rating for wire is much lower than its full potential, 15 amps going over a 10 gauge wire will make it very hot.

Every material has a temperature coefficient that shows the change in resistance over temperature. I will use the $1 \mathrm{~m}$ of 20 gauge of copper wire and a $50 \Omega$ carbon resistor to show how temperature can affect their mathematical representation.

$$
\Delta R=\alpha^{*} \Delta T
$$

$\Delta R$ is the change in resistance, $\alpha$ is the temperature coefficient, and $\Delta T$ is the change in temperature

$1 \mathrm{~m}$ of 20 gauge wire $=33.281 \mathrm{~m} \Omega$ : (Copper's coefficient is $4.29^{*} 10^{-3}$ )

We will assume $20 \mathrm{C}$ as our starting point and show the resistance value at $85 \mathrm{C}$. $\Delta R=\left(4.29^{*} 10^{-3}\right)^{*}(85-20)=0.27885, R=R_{0}^{*}(1+\Delta R), R @ 85 C=42.561 \mathrm{~m} \Omega$, this is $25 \%$ increase in resistance.

For a $50 \Omega$ Carbon resistor:

$\Delta R=\left(-4.8^{*} 10^{-4}\right)^{*}(85-20)=-0.0312, R=R_{0}^{*}(1+\Delta R), R @ 85 C=48.44 \Omega$, this is $3 \%$ decrease in resistance.

Some applications require precision values for various reasons and the values represented demonstrate why application dictates design and materials. Though most electronics will never see temperatures of $85 \mathrm{C}$ it is usually the maximum specified temperature for a lot of applications. In the case of precision electronics, especially in analog technology, these deviations in value need to be controlled or have an allowed tolerance for error. Even though some components can be manufactured to within $0.00001 \%$ of the specified value or better, environment and use can alter precision no matter what, this is very important when it comes to electronic reliability because 
hundreds to millions of components can be used in an electronic system and any one system can affect the others.

An inductor is another form of a discrete component. The purpose of inductors range from radio frequency (RF) chokes, power supply rectification, and power transformers (the process of converting AC to DC electricity). An inductor simply is a wire wrapped around a magnet combining conduction and magnetic flux. An inductor does not have a direct current resistance level in ohms but contributes to system impedance, impedance is a set resistance based on frequency.

Inductors gain in impedance levels when the frequency increases; this is why they are used as radio frequency (RF) chokes because they can reject certain frequencies based on design, though inductors are generally used to reject higher frequencies. Inductors are also used in power supplies to control the flow of electricity and help prevent any undesired effects such as ripple current. Inductors are generally used in analog circuits for the purpose of blocking AC or signal energy and allowing DC to pass. Inductors are a robust component but are not immune to deviations as wires and resistors are. Environment and current flow affect the values on the device, take the wire deviation mentioned above along with changes in magnetic flux over temp humidity, etc. a tolerance needs to be stated for practical design in precision electronics or power supplies.

When it comes to wires, resistors, and inductors they have a long life span due to being a solid material and studies in reliability generally focus on handling power and accuracy and in the case of inductors the life of the magnetic flux. Since all three of 
those components allow DC current to pass controlling and rating power usage is important as to not destroy them or shorten their lifespan.

Capacitors are another form of discrete component but unlike the previous components mentioned capacitors are very volatile component and can fail for a wide variety of reasons. The study of reliability of passive and discrete components has the most focus on capacitors.

A Capacitor is a component that stores a charge and discharges in set intervals at specified voltages and in some cases specified frequencies and polarities. The impedance rating for a capacitor goes down at higher frequencies, the only difference is that it goes down at a non-linear rate. At $\mathrm{OHz}$ which is the operating frequency of $\mathrm{DC}$ current its resistance is supposed to be infinite, in other words a DC block. However this is only an ideal situation, not a real one.

There are many designs of capacitors but the simplest one to explain is the parallel plate capacitor. It is two conducting plates separated by dielectric material. A dielectric is an insulator designed for a specific purpose which is usually rated to allow electrical fields to move in a certain manner. A common example of a dielectric is seen in coaxial cables, the purpose of that dielectric is to confine the signal energy to the solid core of the cable to prevent signal loss and to separate the core from the shield outside of it. As stated dielectrics can be designed to pass a certain charge or reject it, this is what separates a dielectric from what most people perceive to be a common insulator. 


\section{CHAPTER 3}

PARASITIC EFFECTS ON ELECTRONICS

In design and learning concepts of electronics we learn them by understanding the ideal situations. In the real world there are many parasitic effects or limitations that do not allow ideal or designed specifications to work. Additional testing and design is required to achieved a desired effect.

Some of the issues that surround overall device reliability and accuracy are:

- Leakage current

- Noise

- Ripple Current

- Temperature

- Environmental effects

- Design

Leakage current is one of the most common issues in electronics. It is very undesirable for many reasons. One reason is that in circuit design there are points in the circuit where DC current is unwanted, this is one reason why capacitors are used as DC blocks. Another reason is that in some circuit designs, an op-amp is one example, no current is to enter or leave it to properly amplify the signal energy introduced. Leakage current can also shorten battery life, batteries have a set usage rating and will remain active even when the device is turned off. When current is allowed to leak through various components the charge in the battery will find a route to ground even when the device is turned off, hence leakage. Though the effects of leakage current are minimal in the short run and will not immediately cause failure, in the long run and in the 
terms of device reliability it is a very important issue, especially when devices are specified to last for at least 10 years or more.

As mentioned before capacitors are one of the most volatile discrete or passive components in circuit design. This is because of the dielectric materials needed to insulate DC current but allow signal energy to pass. When DC current passes through a dielectric it causes the composition of each dielectric section to deteriorate over time. Dielectrics are not only used in capacitors but also in semiconductors and any other application where DC current and Signal Energy needs to be controlled.

There has always been the myth that when you leave the power on in any given system it lasts longer and performs better, to most people this sounds like a myth but it is actually true. A study by Jens Both from BC Components in 2001 published an article which shows the test results of automotive grade electrolytic capacitors on their leakage current and properties under different conditions. The study concerns the 148 RUS Series but the same idea covers most capacitors.

The Figures 3.1 to 3.3 below show the tested results of leakage current over time and temperature: 


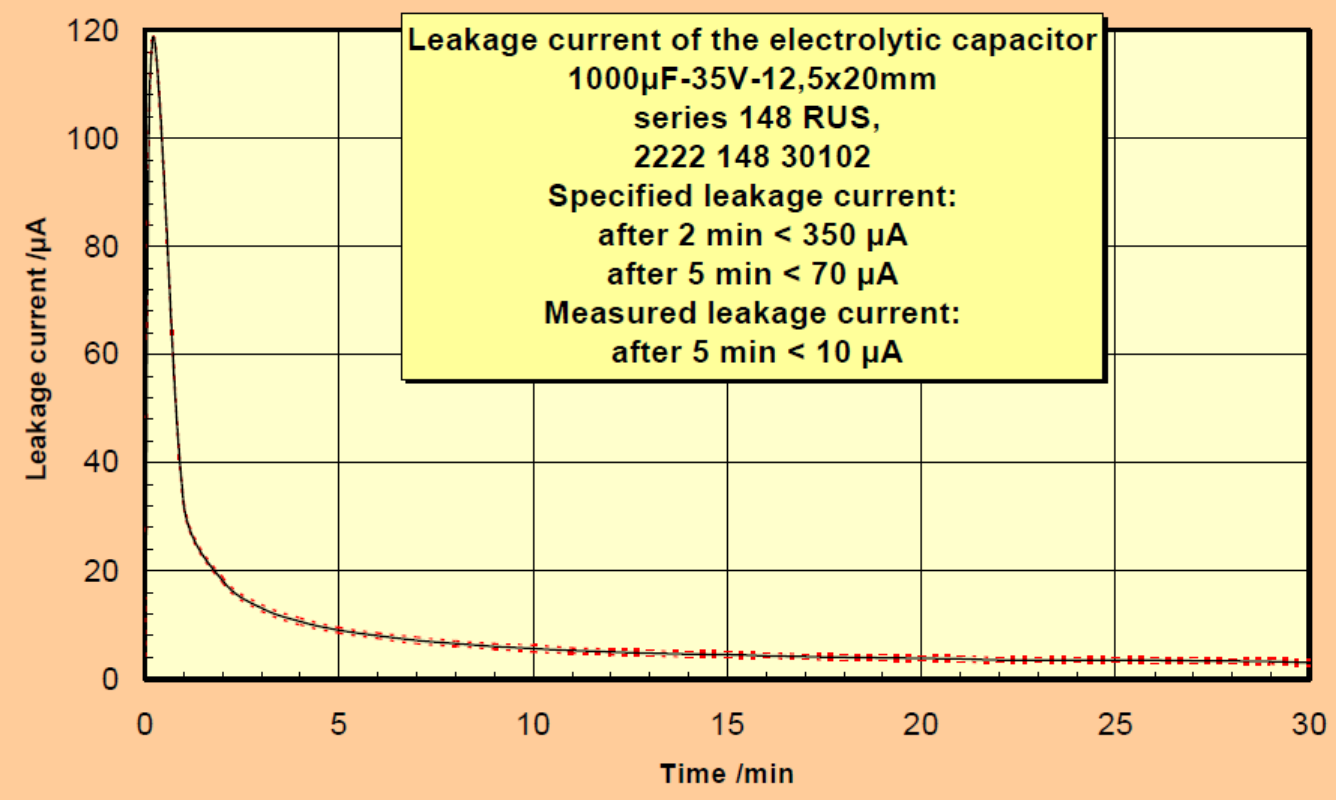

Figure 3.1 Leakage current over time.

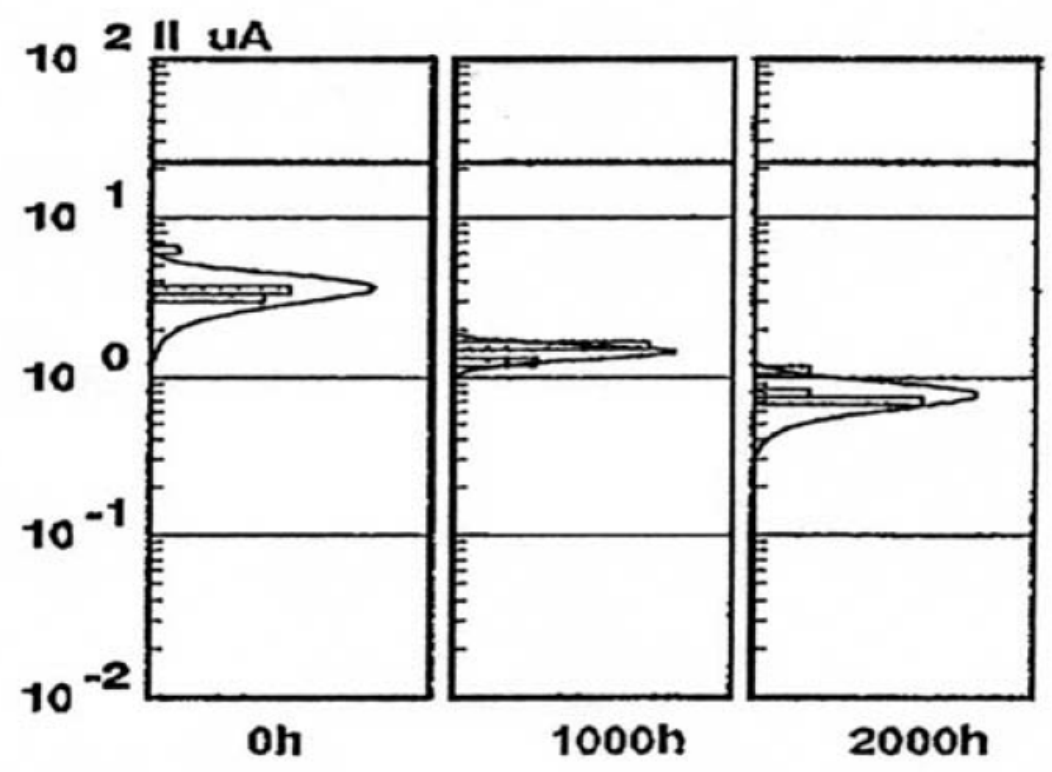

Figure 3.2 Leakage current over extended periods of time with statistical distribution. 


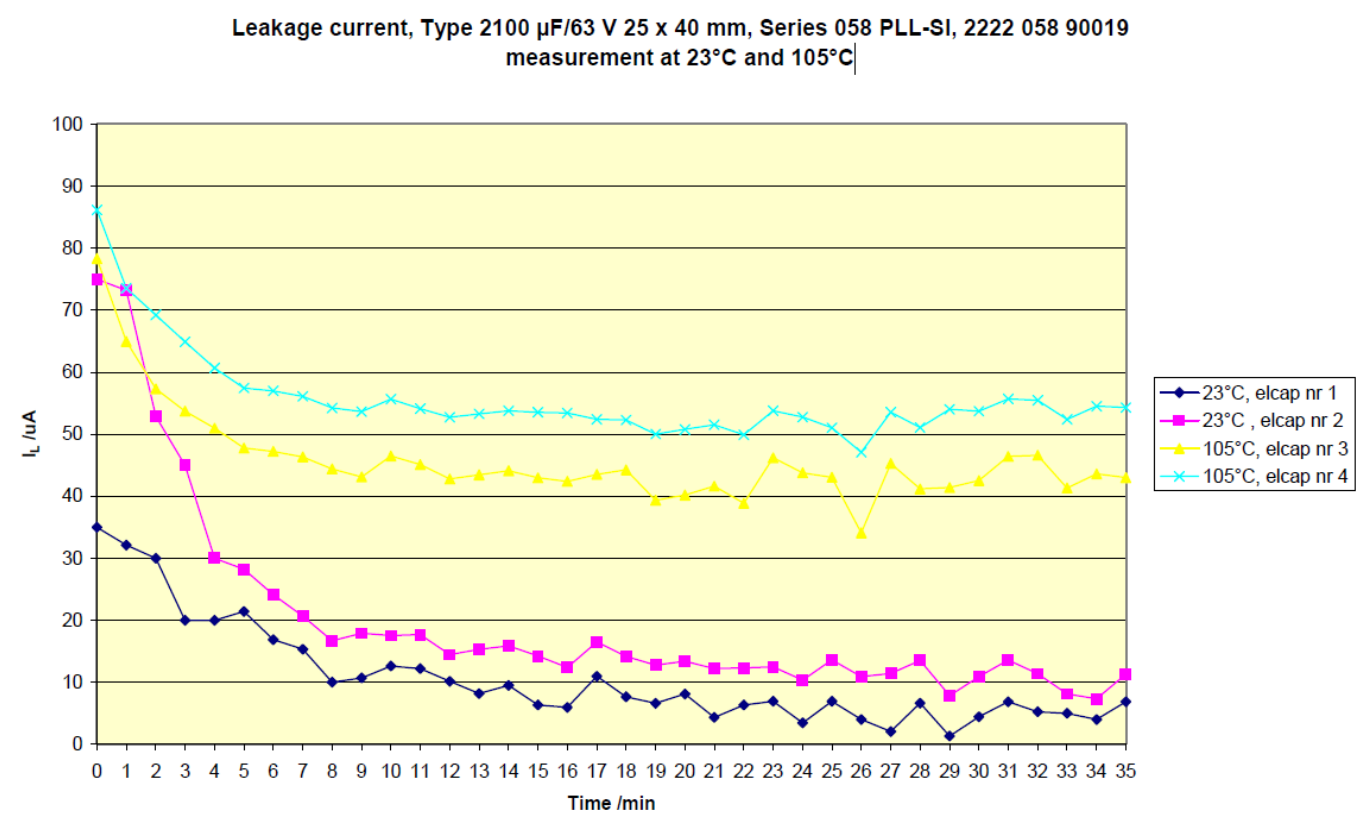

Figure 3.3 Leakage current over time and temperature.

Allowing more current to go through over temperature can be more detrimental to the circuit or system depending on the application. Where temperature can cause a major issue with leakage is in terms of breakdown voltage. As it is known breakdown voltage is the minimum voltage that causes the dielectric insulator to become electrically conductive, this defeats the purpose of the operation of a capacitor and can cause a major failure in a given system. When current flows through a dielectric it can heat it up and cause damage to the insulator on top of the outside heat already applied. A dielectric designed for use in a capacitor or on a semiconductor needs to be specified for a certain operation so in some cases modifying a dielectric is not possible.

Most manufacturers test breakdown performance over that of the specification to insure over voltage protection to prevent the capacitors or semiconductors to fail. Below is an image of high voltage dielectric breakdown of a piece of Plexiglas: 


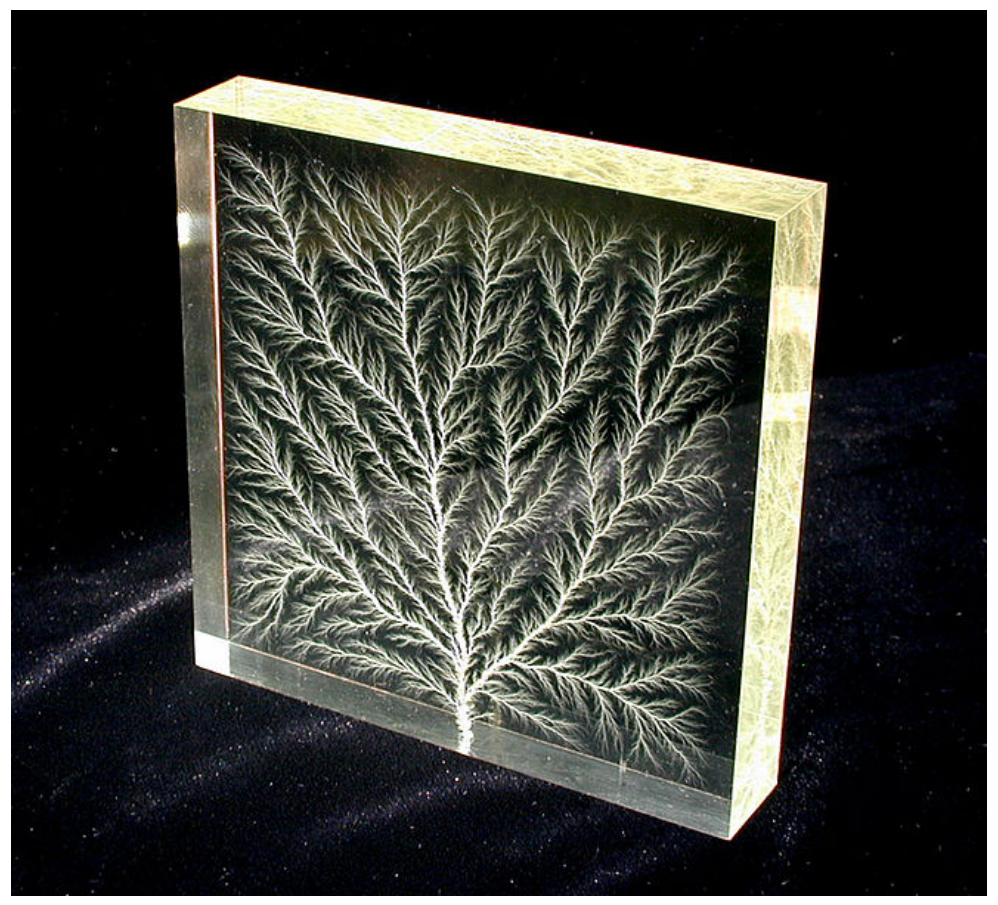

Figure 3.4 Plexiglas after breakdown voltage limit was applied and reached.

This is an extreme case but it helps demonstrate what happens to the dielectric structure should it breakdown and fail meaning allowing current to pass. This is a visual demonstration on why leakage current, temperature, voltage and other controls need to be in place.

To get to a valid point on a side note, these controls are extremely important in mobile devices which only allow room for a passive cooler and where battery life is extremely important.

Over time leakage current passing through the dielectric structures causes them to decay be means of structural integrity. That along with the temperature coefficients causing dielectrics to become more conductive at higher temperatures can cause major problems with the device and its system or a complete system failure. Controlling the outside environment is one way to curb the temperature issue but there is always the 
actual temperature of any given component and in a sealed imbedded system like a smart phone these issues must be accounted for.

As it is commonly known when any material is repeatedly heated and cooled its structure decays. This along with voltage applied can further weaken any capacitor. The best visual example I can give of a capacitor in motion is a picture taken from Wikipedia based on the Hydraulic Analogy in physics shown below:

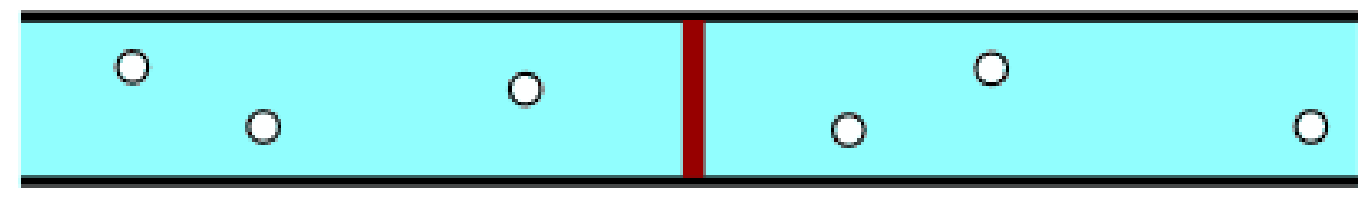

Figure 3.5 Illustration of the hydraulic analogy (red diaphragm moves from side to side).

The read diaphragm in the middle is the dielectric and it moves based on charge, when it curves one way and remains there it is a good explanation on why leakage current goes down over time, the dielectric structure is adapting to charge applied. The voltage applied controls how much it shifts, I wish the animation would remain in the word document, if it shifts to far the structure will collapse causing failure like in the Plexiglas example above.

Some ways of controlling dielectrics can be material purity to insure that any impurities present do not cause parasitic effects. Another way is the choice of materials, some capacitors use liquid or solid forms of dielectric or conducting material and in some cases polarity. Eventually it comes down to cost and what the customer is willing to pay for in any given system. For the consumer absolute accuracy is not always 
required and the life expectancy for a consumer device can be 5 years in contrast to corporate or government usage where they want a 20 year or more life span. This is where cost and choice of materials can be an issue.

Signal noise is a major issue in both wired and wireless technology, this is why many capacitors, inductors, etc are used for input and output circuits. As stated above it is important to have controls on the components and devices itself but how do you make it where you can accept a wide frequency range? In a lot of circuit designs I have seen over the years there are usually 5-10 capacitors, and in some cases a few inductors, in use to filter out unwanted frequency ranges. They are usually different capacitor values attached from the input or output line in parallel to ground to reject higher frequencies.

Why reject higher frequencies? In the case of signal noise, when noise is introduced into the signal for various known reasons, and some unknown, they are usually much higher frequencies introduced to the desired frequency and as stated before capacitors allow for passing of higher frequencies. Depending on the application signal noise can cause a lot of error to an application and in the case of today's high speed data requirements it can be a major issue. 

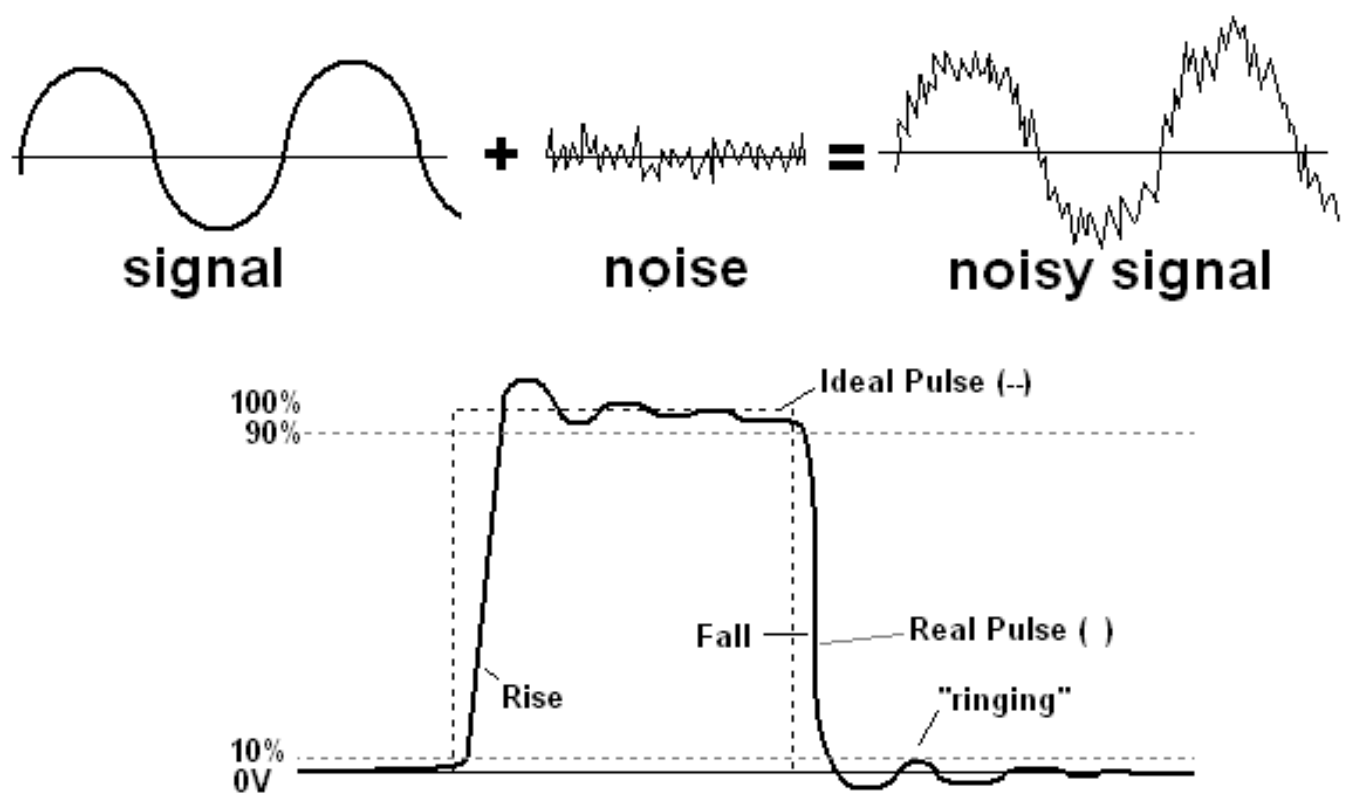

Figure 3.6 Ideal vs noisy waveforms.

Notice how the noise is a higher frequency than the source in the case of both analog and digital signal? In the case of lower frequencies it is very easy to filter out the noise but with today's and future requirements of high speed and higher frequencies the frequencies being used are getting closer to that of the noise that exists. This is one of the reasons for the switch from analog to digital technology but I will cover that later on.

Signal noise can be suppressed to an extent with discrete and passive components. Two basic examples are high pass and low pass filters. The images I am providing are extremely simple examples but should show how signal suppression works: 

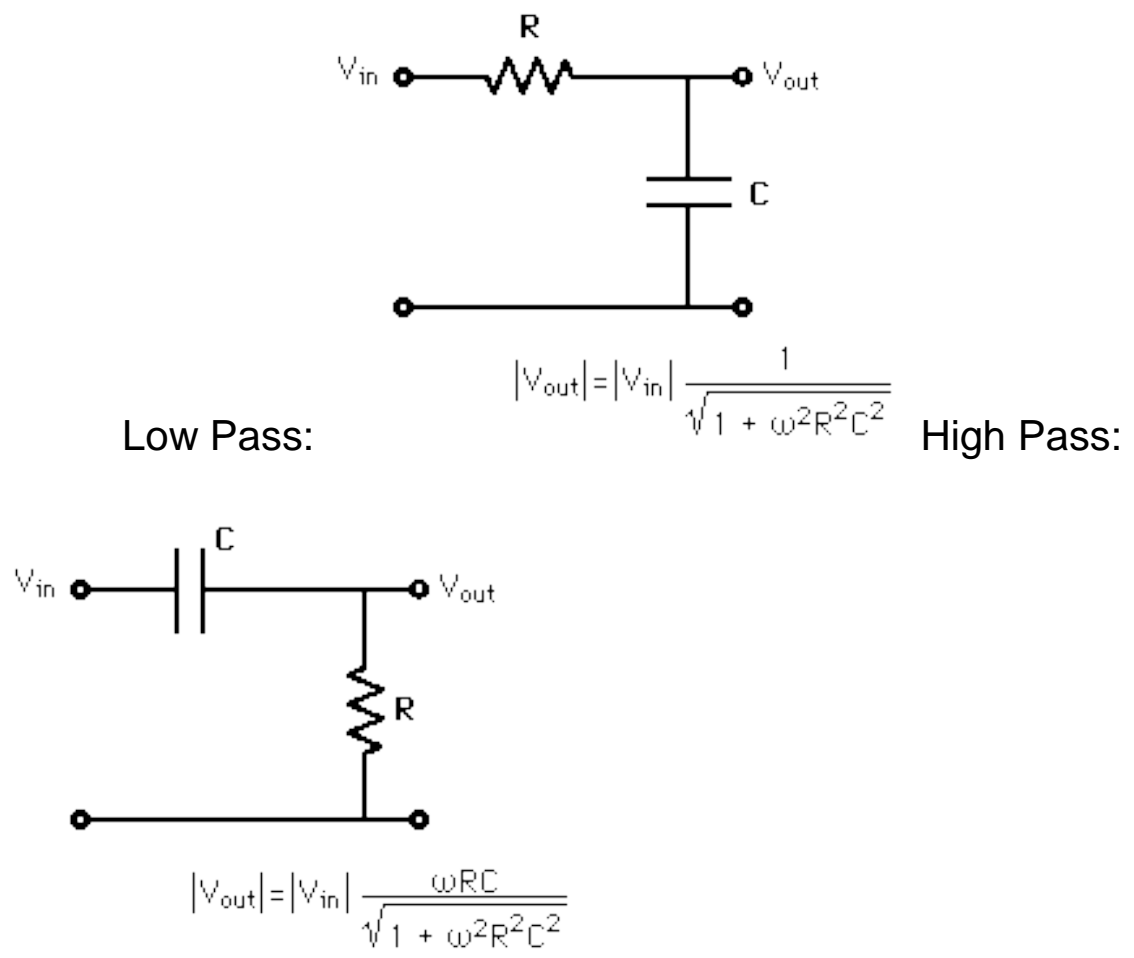

Figure 3.7 High pass and low pass circuit examples.

The capacitors in the circuit either serve to send higher frequencies to ground or stop low frequencies to enter the output. In the event of a capacitor failure in a low pass circuit higher frequencies and noise will enter the circuit causing a failure or in the event of a failure in a high pass circuit the circuit will either short out or conduct to the output allowing DC current to pass or lower frequencies to enter. Both cases can cause a system failure.

Ripple Current is another form of noise but it is more focused on power supplies. When an $A C$ power source is converted from $A C$ to $D C$ the output voltage and current is supposed to be $0 \mathrm{~Hz}$. When ripple current is present this is not the case causing variations in the DC voltage, this can cause over or under voltage which can damage electronics.

Source: http://www.allaboutcircuits.com/worksheets/supply1.html 
The images below show what happens when an $A C$ circuit is rectified and converted from $A C$ to $D C$ with and without filtering:
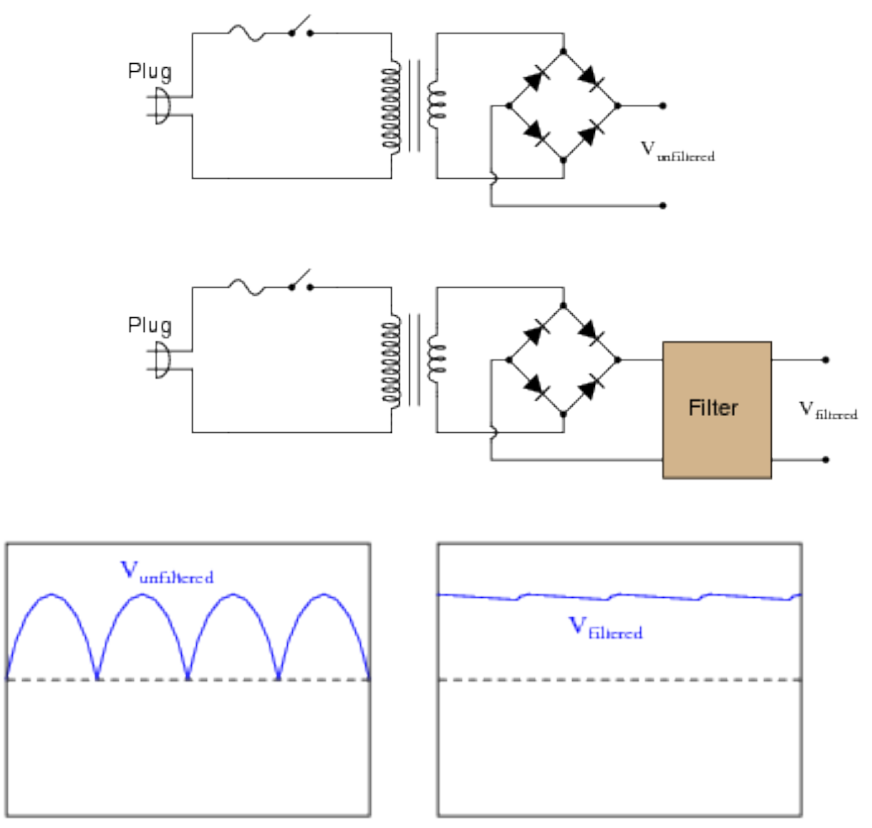

Figure 3.8 Rectified AC waveform.

The filter design is what makes or breaks the quality of the DC current supplied. On the graph DC current should be a straight line. Some applications can get away with a set DC voltage say $+/-10 \%$ but some cannot, it all depends on the application.

For applications like microelectronics next to no ripple variation can be tolerated because semiconductors require a set voltage to be active or inactive and with modern technology the difference between on and off is now as low as $1.0 \mathrm{~V}$. When a higher level of precision is needed various discrete components, voltage regulators, etc are used, what separates the good from the bad power supplies is the quality and variance control of the power supply. 


\section{CHAPTER 4}

\section{COMPONENT RELIABILITY}

Reliability falls under the realm of Quality Control, the question is how do you make a device more reliable or guarantee a lifespan? This usually falls under production engineering once a design of a new component or device is complete. A design in itself may work under simulation or controlled circumstances but it does not necessarily work as intended out in the field for the intended customer.

In terms of hard data most companies or other agencies will not release any of their important testing or re-design results and consider anything that gives them the edge over their competitors a trade secret and protect it under law. It is not difficult to figure out how a device or component works but it is not easy to figure out how a manufacturer made it possible. The only thing that can be explained from the outside in a research capacity is explaining methods to help increase lifespan, specification control, and reliability.

The first step in component quality is the choice of materials. As stated before I chose copper wire and carbon resistors to show temperature coefficients but those materials are not always desired for every application. The reason why I chose them is because they are common and inexpensive. That leads to the next question, why not choose the best material for everything? This is because the best materials may cost more than the customer is willing to pay and may have parasitic properties under certain circumstances. The price for any material will change over time, increased demand or a shortage can cause increased prices. 
The choice of materials for wires in the electricity and electronics realm is basically common sense but I want to take some common examples of the known best conductors to explain why someone would choose one over the other.

As far as resistors go every manufacturer has a specified resistance level, resistance variance (tolerance), and wattage. Most modern electronics are run on a printed circuit board PCB and therefore require a small resistor is needed in the design hence chip resistors are used. By way of design the best way to help insure that a resistor does not fail is by not exceeding $50 \%$ of the rated wattage. Why not use a device at its max rated wattage? Because running any electronic device at max capacity will shorten its life span by either a material breakdown or degradation of material structure. Manufacturers over watt the device at test to guarantee safety, drift, and life testing for any given component or device but just because it can pass a test doesn't mean that it should be used at max levels at all times. Also various studies have shown from power supplies, discrete components, etc that electronics in general has peak efficiency at $50 \%$ load.

Most chip resistors use thick film, thin film, or resistance chains inside the chip package, the name alone can give an idea what they might be used for. In a published article by Panasonic, a known manufacturer http://industrial.panasonic.com/wwwdata/pdf/AOA0000/AOA0000PE36.pdf (found here), they explain some uses of their resistors and methods of attempting to force a failure and shorten its lifespan.

Application of thin film resistors is more widely used in electronic systems, this is because they present less noise and can provide higher accuracy. Thick film resistors are more used in power supply applications. This is because they can allow a higher 
amount of wattage to pass and can be used for low resistance levels. Chain resistors are a bit harder to explain because chain resistors are used in a network inside the package either in series, parallel, or both.

The choice of resistor depends on application but in the realm of electronics (excluding power supplies) thin film is the most ideal. In all the failure scenarios presented in the article the thin films resistors have an end result of an open circuit, this is important because if designed correctly an open point in the circuit will cause the system to stop and shut down. In the case of thick film an open circuit is not the only failure scenarios create an open situation making predicting reliability very difficult. The resistance value of thick film resistors can change with overvolting or a large enough surge in power. A change in resistance value can cause a major failure in a system because any given design is dependent on knowing the possible range of all components used for resistance, noise, and power rating. When an overvolt or overwatt situation occurs it is unknown on what the specification of a thick film resistor will be. That and allowing current to pass can cause degradation to any given system. The study is focusing on electronics so I will focus on thin film resistors

The choice of materials is another way to insure component reliability and accuracy. In the case of thin film resistors Nichrome ( $\mathrm{NiCr})$, which is about $80 \%$ nickel and $20 \%$ Chromium, is a common material used. Nichrome is commonly used due to its reliability, low noise, easy control, and relatively low cost. Accuracy is controlled in a few forms, as described earlier temperature can affect resistance values, Nichrome has a coefficient of 0.0004 on average in pure material form. As we know insulators have a negative temperature coefficient and conductors have a positive one. Nichrome has 
been widely used for over 100 years because of its properties of temperature stability and oxidation resistance, this makes it an ideal choice for use in electronics.

For a $500 \mathrm{hm}$ Nichrome Resistor at $20 \mathrm{C}$, the resistance at $85 \mathrm{C}$ would be $51.30 \mathrm{hms}$, that's a $2.6 \%$ variance, which doesn't seem like much but in microelectronics it can be a lot. Another way is to cut the resistor to a desired value, this can be done by laser trimming, by micro machining the resistor to value, or by controlled depositing of the material.

A second material worth mentioning is Sichrome ( $\mathrm{SiCr})$, this material is less documented because these resistors are usually made on a wafer through material deposition. There is little data about SiCr because every manufactuer has a different method of making the resistors and have multiple patents concerning it. The TCR of Sichrome is roughly -0.0004 , in combination with Nichrome in a chain formation this can make an ideal situation with offsetting temperature coefficients. This is commonly done with semiconductors and discrete components made through wafer processing or other silicon manufacturing methods.

Since a wafer is pure silicon, chromium can be introduced to it to create SiCr. Through manufacturing methods the TCR, size, resistance level, etc can be easily controlled. One example would be:

US Patent: US 6171922 B1, found here: http://www.google.com/patents/US6171922, published in 2001 and filed by National Semiconductor. This explains a process of controlling the TCR through wafer fabrication processing. There are many other patents out there like it, since SiCr can be made through chemical deposition many companies and organizations do not reveal the data of SiCr resistors. There are few physical tables 
out there about it because it can be varied by process control, the idea is to either keep it close to 0 or use it to offset the TCR of all other materials used.

Resistor manufacturing is a challenge in itself but it is easier to control than other components. Size, material purity, material choice, material behavior, and process technique are even more critical when it comes to capacitors.

In semiconductors and microelectronics, resistors and capacitors are the most commonly used discrete components, especially inside an IC. Diodes, transistors, etc are also main building blocks but I will be getting to those soon.

There are three commonly used capacitor types used in electronics: ceramic, film, and electrolytic. Ceramic and film capacitors are generally non-polarized and easier to produce, both of these are solid materials and are easier to manufacture and control. Electrolytic capacitors can come in solid and non-solid form, the capacitors are desired in cases where polarity is needed and where a high capacitance level is needed. The picture below shows the capacitance and voltage where types of capacitors are needed: (Source: Wikipedia Images) 


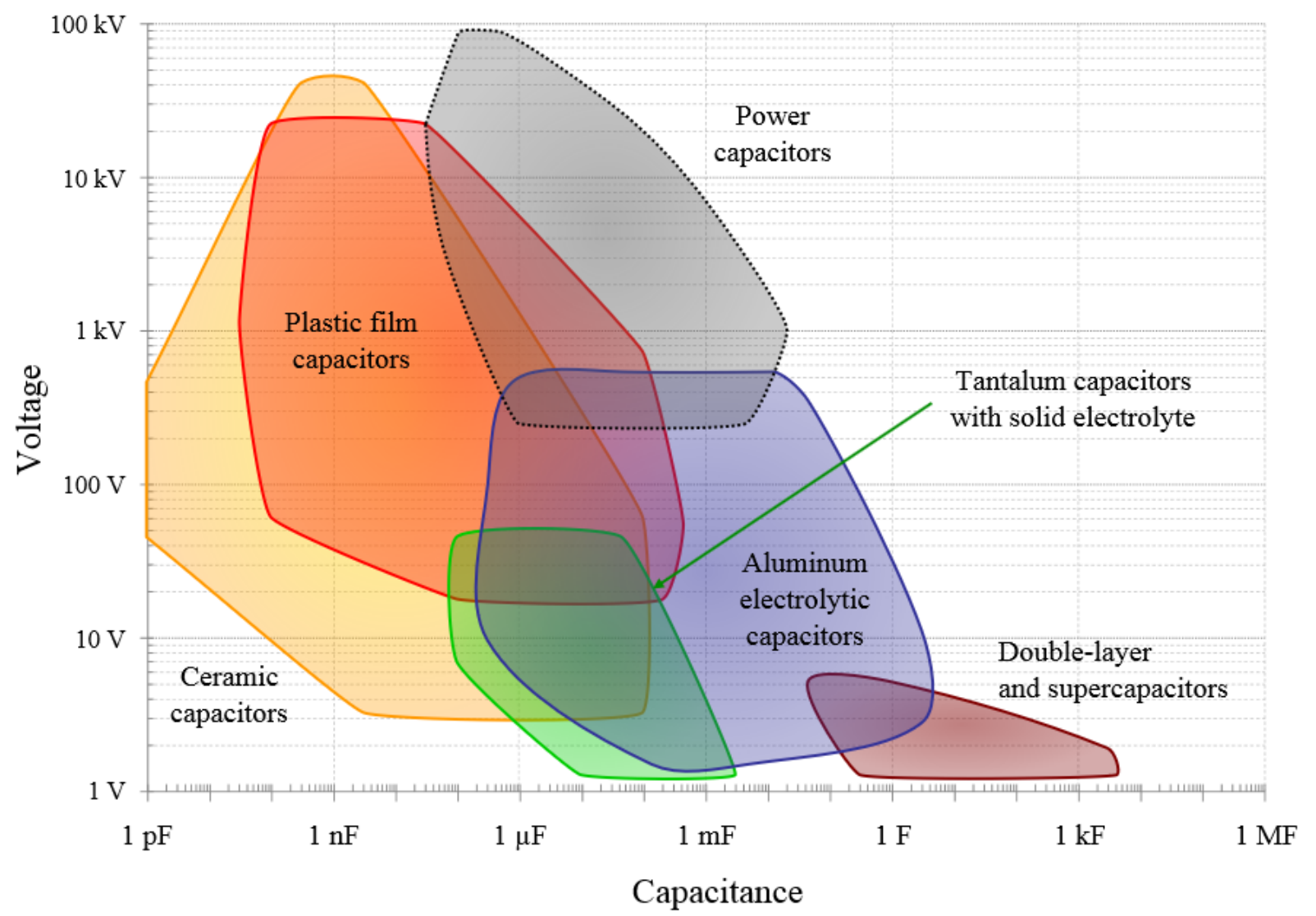

Figure 4.1 Capacitor types and typical values.

A system can be designed for the best possible level of efficiency based on customer specifications but the question is will the components being used hold up to the task? The original design of a parallel plate capacitor pictured below: (Source Wikipedia Images) 


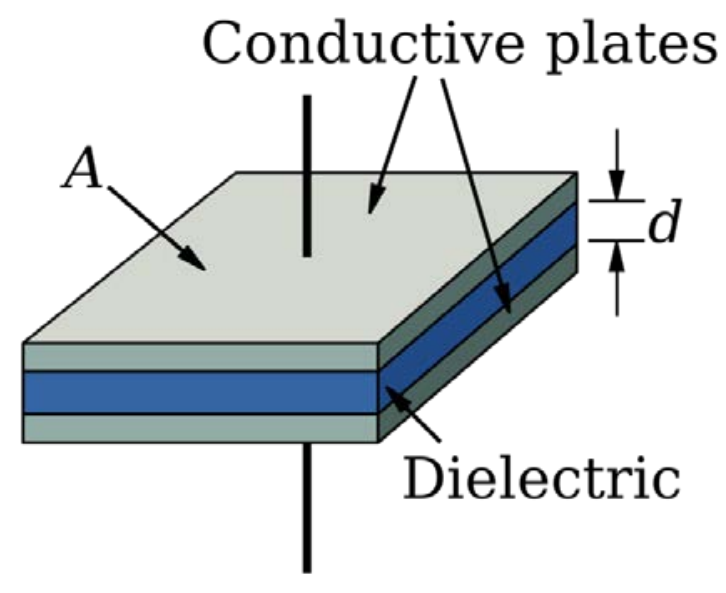

Figure 4.2 Simple parallel plate capacitor design.

This is the simplest capacitor design but if the dielectric fails or breaks down physically then the entire capacitor fails. As mentioned before keeping control on the breakdown voltage is easy to do provided the initial tests can confirm performance. The material purity is another control to insure reliability. When defects are present in the dielectric it can cause a lower lifespan because the dielectric constant calculated by design will be skewed or it can cause premature breakdown of the structure.

The ceramic capacitor is one of the most common types of capacitors used individually, because they are inexpensive and easy to manufacture. There are many classes, subclasses, and doped materials used for each type for a given application which can range from filtering, coupling, bypassing, or charge volume. 
Figure 4.3 below provided by TDK :

http://product.tdk.com/en/environment/ecolove/eco18000.html

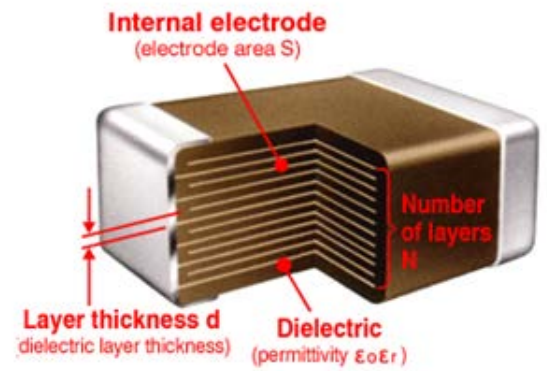

Capacitance $C=\varepsilon_{0} \varepsilon_{r} \frac{S}{d} N$

C : Capacitance

$\varepsilon_{0}:$ Permittivity in a vacuum

$\varepsilon_{r}:$ Relative permittivity of dielectric

$S$ : Electrode area

d : Dielectric layer dimensions

dielectric layer thickness) (permittivity $\varepsilon_{\circ} \varepsilon$,

$\mathrm{N}$ : Number of layers

Figure 4.3 Multi-layer ceramic chip capacitor cross section.

So far the issues of temperature and power have been spoken multiple times.

The reason for this is because temperature, power, charge, etc are all forms of stress on any electronic or electrical device and the situations need to be controlled for a system or device to meet specifications.

Extreme cases like improper soldering, high wattage, or high temperature can lead to defects seen in figure 4.4.:
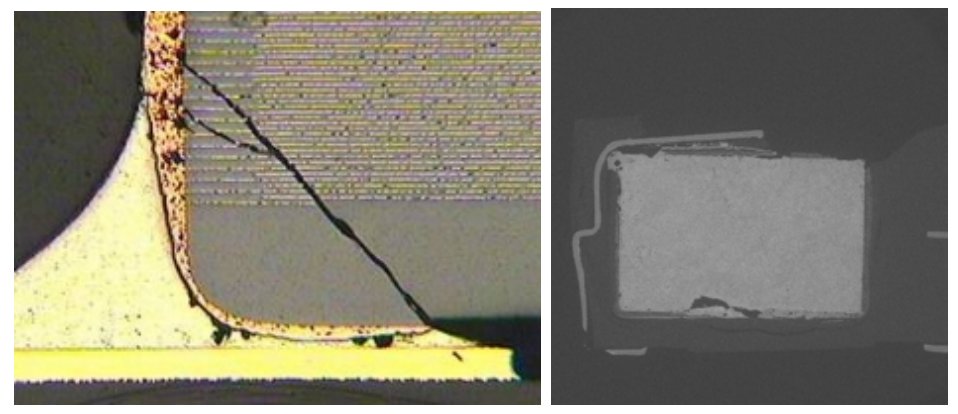

Figure 4.4 Defects of chip capacitors due to high temp or high wattage.

http://www.nextekinc.com/precision_micro_sectioning.html(Source1)

http://www.semlab.com/examples/crosssections/crosssections.html (Source 2)

Those are cases that can cause complete failure or extreme capacitance

changes. Since electronic applications like smart phones contain over 300 capacitors 
outside of IC packages the size of the capacitors need to be very small. Through modern manufacturing techniques smaller size chips maintaining the same capacitive values has been possible but at the expense of added number of electrode layers and decreased dielectric layer size.

It is possible to control the dielectric constant as well based on material and dopant concentration. As described in the formula above there are multiple trade offs that can be used to maintain the same capacitance value. Since reduced size and increased performance is always the objective what can we control to make this happen. First is making sure the electrodes and dielectric areas have no flaws. Excluding the crack from the image above notice the black specs in the electrodes and mild variation in color in the dielectric area? These can be anything from a process bubble from manufacturing to an impurity. Either of these situations can skew the dielectric constant and flow of capacitive charge and must be controlled. This is where advances in manufacturing technology come in. To grantee less flaws increases the cost but also increases reliability. Most importantly the reduction in flaws helps prevent leakage current.

As for accuracy over temperature, there are different classes of ceramic capacitors used.

Class 1 capacitors are known to be the most accurate over temperature, these are the ones generally used for noise filtering. Manufacturers can guarantee these to have as little error as less than $1 \%$ change from $-55 \mathrm{C}$ to $125 \mathrm{C}$. The trade off is high accuracy capacitors generally have a low dielectric constant and must have low capacitive values and voltages as a result. For lower capacitances they have lower 
impedances at much higher frequencies so for high speed and frequency applications these are an ideal choice. For low frequency and high voltage applications the opposite is true, this is where class 2 MLCC's come in. Though they cannot maintain the same accuracy over the same range they can maintain between $10-15 \%$ accuracy over the same temperature range and have a higher dielectric constant, making them easier to manufacture and cheaper for the customer. The choice of which to use when is up to the designer.

Film capacitors are another type, where these are important is that they not only exist in exterior packages but are also used in fabrication on IC chips if a capacitor is needed inside the package.

A bit of feed forward, IC chips generally have transistors, diodes, film capacitors, and film resistors inside the package. This can be done because in wafer processing multiple layers of metal conductors, dielectrics, and doped silicon are used and those devices can be created on wafer easily if needed on the IC. Inductors, relays, polarized electrolytic capacitors etc are generally placed outside the package because of the practicality and properties of their use. Research is being done this day to print inductors in an IC and package and relays in an IC via MEMS but these are still in infancy and are possible now but expensive and hard to come by.

Film capacitors are known for their high levels of accuracy both in tolerance and over temp. Tolerance can be achieved by means of wafer fabrication, laser trimming, or processing techniques. Like Class 1 capacitors they usually maintain a low dielectric constant and have lower capacitance values. 
Where attention is usually paid in terms of reliability is in electrolytic capacitors. As the name presents the dielectric is a liquid electrolyte. These capacitors are known to have the largest drift over temperature, highest current leakage, and high variance in capacitive value. Where these capacitors are highly needed is in applications that require high capacitance values and polarity, usually power supplies, high signal energy, and points where the polarity of the charge is important. There are other designs in the mix that do not use a liquid dielectric for the same purpose but they are a work in progress since they cannot yet match the performance or capacitance of electrolytics.

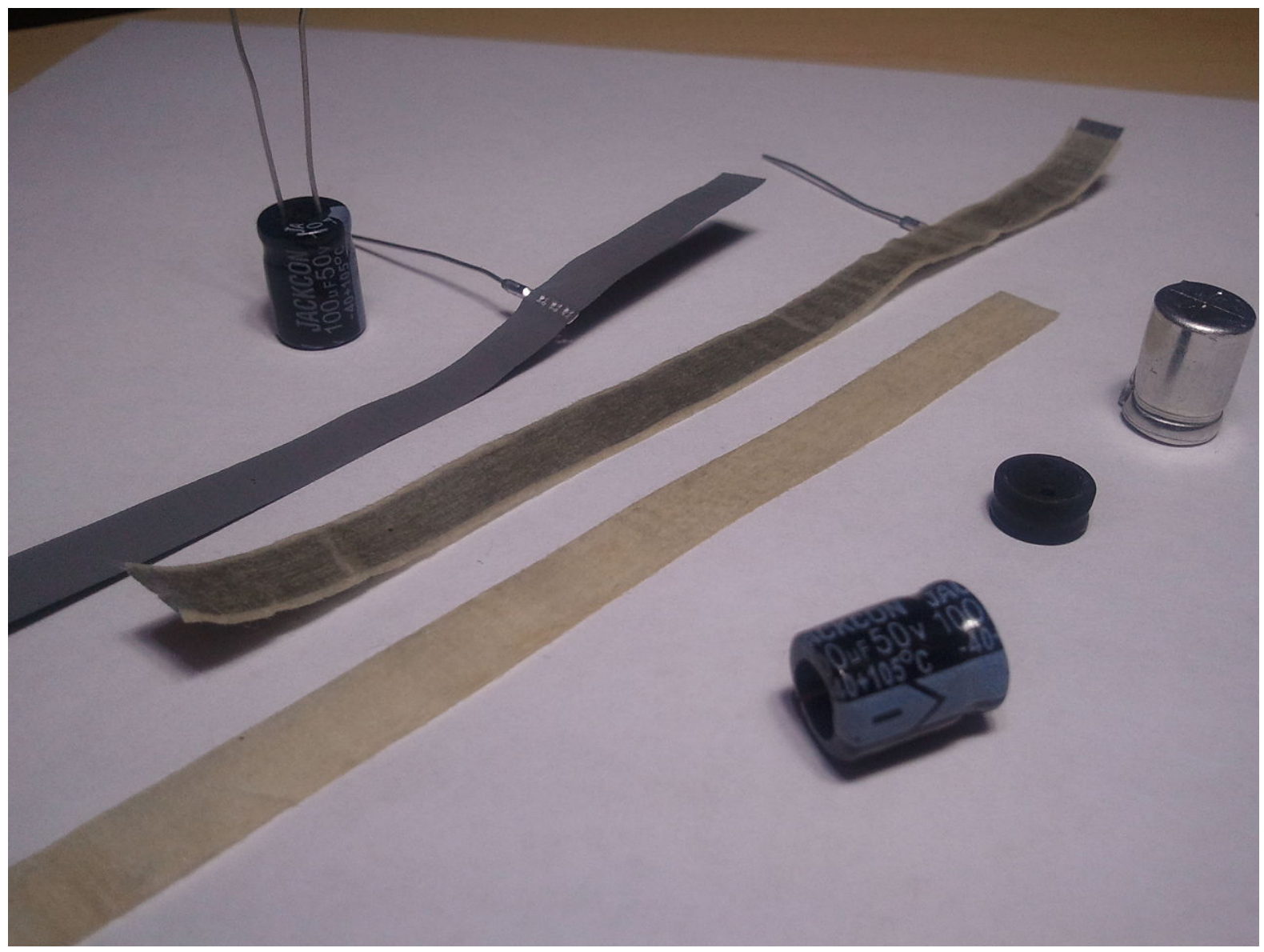

Figure 4.5 Internal components of an electrolytic capacitor. Source: (Wikipedia images) 
Where the reliability of a capacitor matters the most is in the dielectric, over time the liquid will breakdown in its electrical properties. Controlling temperature is a must for these, if the temperature gets too low major current leakage will occur, the liquid will contract causing the encasement to fail (seal break), the aluminum foil can break, or the capacitor will completely shut down. In cases of high temperature the liquid will evaporate causing a possible point of conduction (short), the fluid will expand enough to break the seal of the case, or complete failure of the component. An excessive amount of current, voltage, or wattage can also cause the high temperature fails even if there is temperature control. These capacitors have the shortest lifespan due to usage and the nature of the materials.

The process behind the aluminum fail is also very important. Usually the fail is roughed up on the inside a certain way (manufacturers will not reveal how) before the liquid and paper are inserted, this is to give the charge a direction and control the flow of the charge between the anode and cathode foil.

A reliability article published by Panasonic here: http://industrial.panasonic.com/www-data/pdf/ABA0000/ABA0000TE4.pdf shows various failure modes.

Multiple manufacturers and studies have shows that the ideal temperature for electrolytic capacitors to run is at $40 \mathrm{C}(104 \mathrm{~F})$ this is because it is the optimal temperature for the electrolytic fluids to pass a charge, control leakage, and maintain stability. When current runs through any device it will heat up so keeping the capacitors at room temperature is not practical, In terms of cooling it is much easier to cool to $40 \mathrm{C}$ than 23C. 
The graph below from : http://www.murata.com/englobal/products/emiconfun/capacitor/2012/10/15/en-20121015-p1 Shows accuracy over temp.

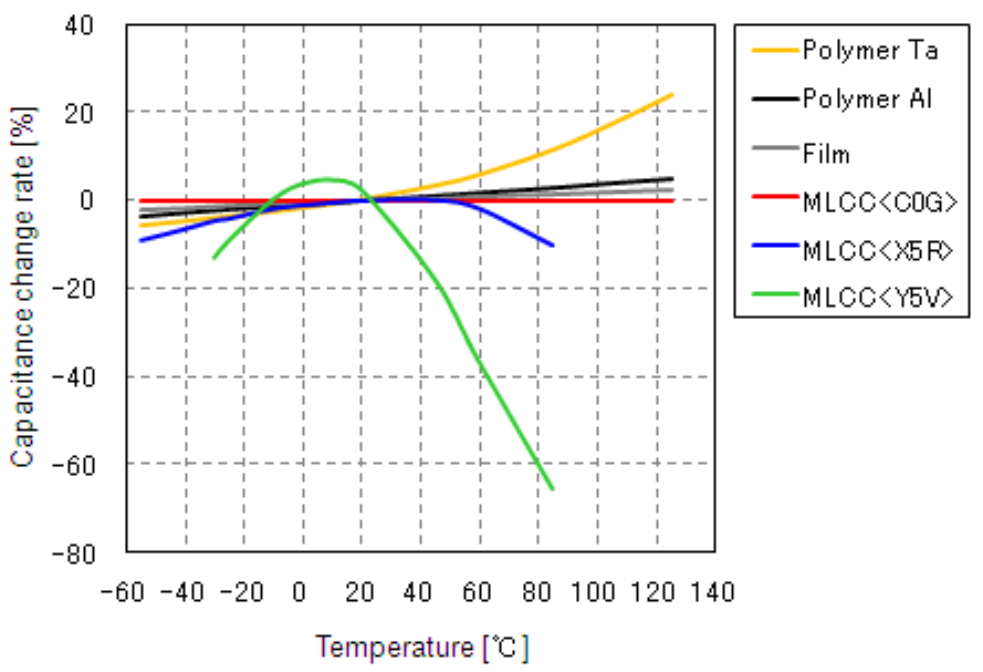

Figure 4.6 Capacitance over temperature.

With exception of the Y5V, a high voltage chip capacitor, 40C is the high point where capacitance values skew off. On a side note this is also why chip capacitors are not usually used for high dielectric constants, high capacitance, or high voltage applications. The Polymer Aluminum and Tantalum capacitors are electrolytic.

Accuracy over temp aside the manufacturing techniques behind electolytics are very important. About 10 years ago there was a major incident across the world called the capacitor plague which cost companies and consumers worldwide billions of dollars because of excess failure rates in aluminum electrolytic capacitors. It is believed that manufacturing processes were either stolen or copied and used to make capacitors at a cheaper price under selling the competition. It was found that the electrolytes had failed prematurely because substandard formulas and manufacturing processes were used. This cost suppliers billions of dollars to honor warrantees and replace the failed devices 
and systems to their customers. The formula for the electrolytes and the foil is a very closely guarded trade secret it is not as easy as copying a method to create a reliable component.
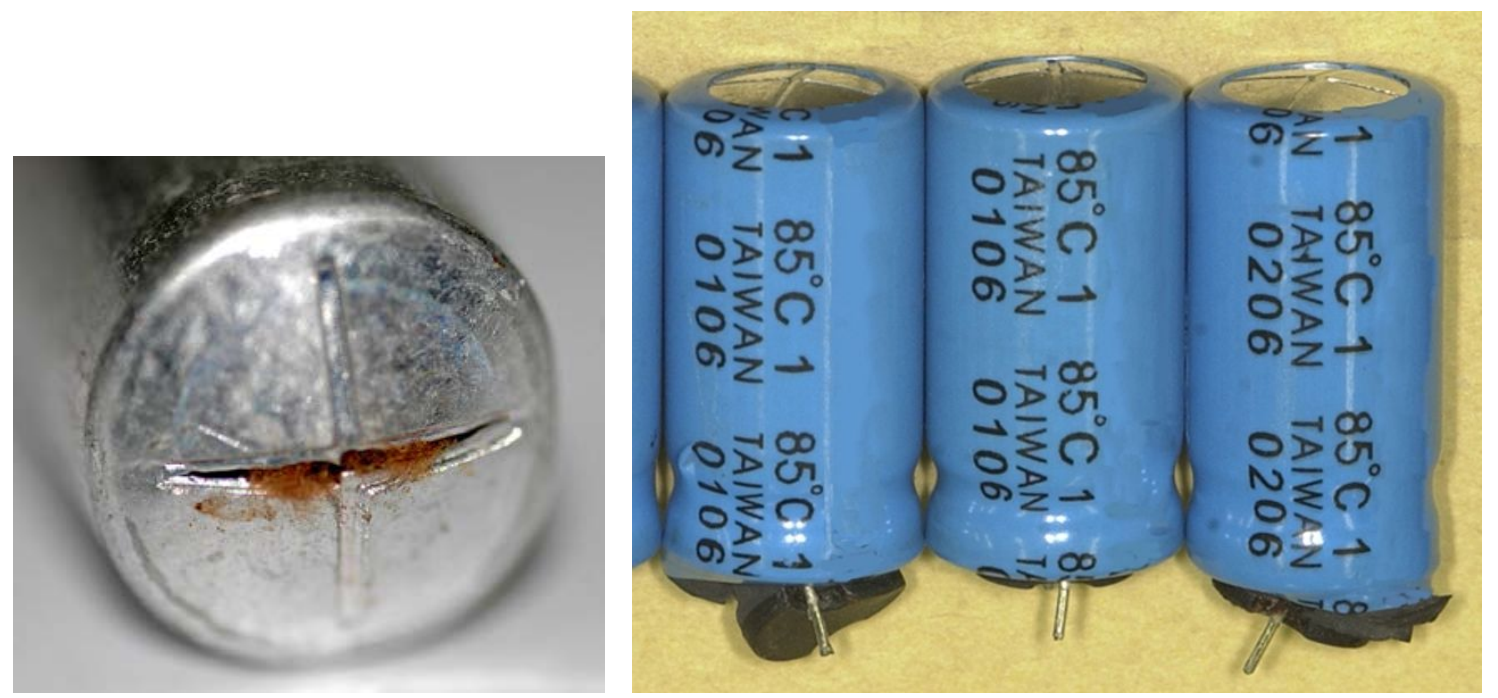

Figure 4.7 Examples of failed electrolytic capacitors. Source: (Wikipedia images)

Life testing is a very important part of determining reliability of any component. In the case of electrolytic capacitors life testing is done in an oven. It is found that for every 10 degrees Celsius (over 40C) the useful lifespan will be cut in half. The graph below shows average life over temp over multiple capacitors used, the component is listed in the graph. Source: http://electronicdesign.com/energy/next-gen-smart-grid-equipmentchallenges-power-supply-designers 


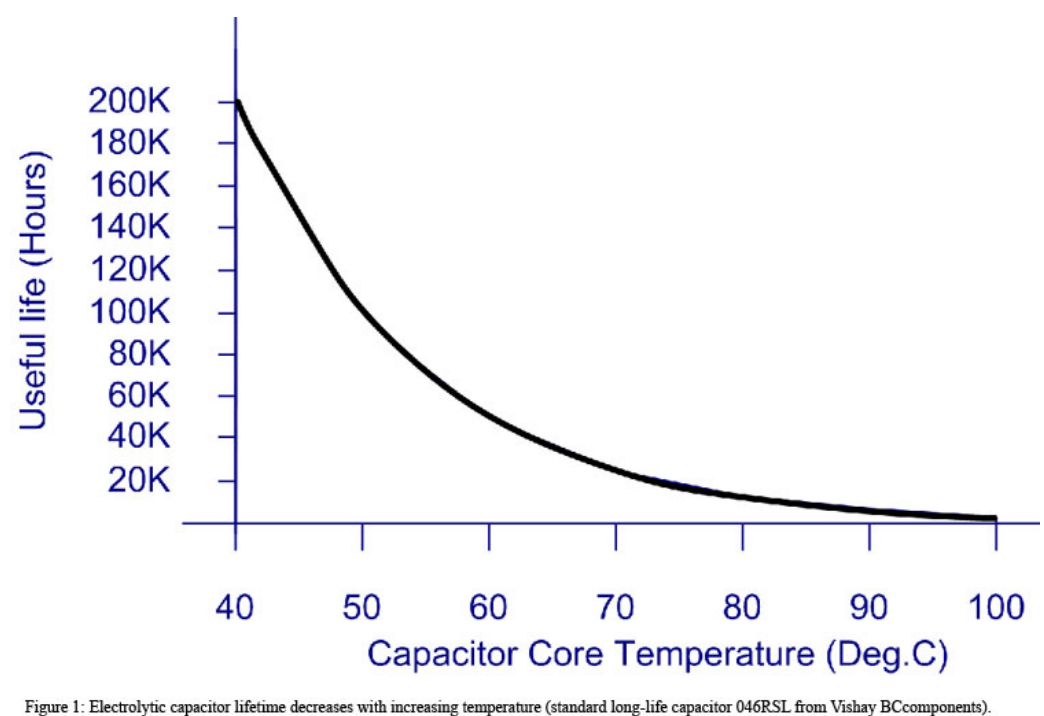

Figure 4.8 Electrolytic capacitor lifespan over temperature.

In the resistor article from Panasonic they ran resistors for long periods of time to determine failure rate over actual use, this was likely done after introducing failures in life testing. In most cases developers and manufacturers do not have 200k hours to run a device, this is why they want to introduce failures early to remove flaws that can be found easily. Once the easy ones are removed then they begin full actual life testing without the added heat or stress, this is why technology development takes up to years in some cases.

For resistors an oven environment is one way to introduce failures but solid components as they are more reliable in most cases have different methods to determine reliability. Shock and stress are usually added to heat, breakdown voltage and over wattage are forms of stress.

Different manufacturers have different methods on increasing reliability, most are considered trade secrets but everyone wants to protect their name and create a level of trust with the customers. The best methods of increasing reliability are choosing proper 
materials, proper design, and high levels of quality control by introducing failures and correcting the errors, none of these are cheap. As none of these are highly discussed in the classroom or a design environment reliability is very important to a company and the customer and is a major field in the realm of engineering, even at the level of discrete components. 


\section{CHAPTER 5}

\section{MATERIALS USED IN SEMICONDUCTORS}

In the process of semiconductor manufacturing the most common base material used is Silicon ( $\mathrm{Si})$, there are multiple reasons why. The first reason for this that it is in the (IV) fourth element group and it is in the middle ground of being either an insulator or conductor. This makes it an ideal candidate for semiconductor usage. By itself it does not act as a conductor or insulator until it is mixed, or doped with the correct materials. It is also the most desired material because it is commonly found, exists naturally, maintains a solid element structure, oxidizes naturally, and is the cheapest to produce. What is used is a refined version of the pure element, the silicon used in semiconductors is extracted from sand and other silicate deposits found on the earth. To make silicon work as a semiconductor it has to be doped with other materials to pass a charge. Boron and phosphorus are two common materials used, they come from the III and V valence groups respectively and make use of silicon's electrical band gap to pass a charge.

Gallium arsenide (GaAs) is the second most commonly used base semiconductor material, it is known as a III-V compound material according to its points on the periodic table of elements. GaAs offers a few advantages over silicon which make it desirable in some cases. The first is that it has a much higher electron mobility than silicon which allows for very high switching speeds and can function at frequencies well over $250 \mathrm{GHz}$. GaAs is also not as sensitive to heat as silicon is, this allows it to operate at temperature ranges outside that of common silicon devices helping to improve device reliability in certain cases. 
As there is much more information about the materials used in semiconductors I would like to get into what can cause non-ideal effects that have to be accounted for in designing semiconductor devices for improved reliability and or non-ideal conditions. Ohm's law as we all know is one of the most basic laws in any realm of electronics whether it be electrical, electronic, or in any other form. As heat and pressure go up so does resistance and since a semiconductor device has an output function based on either input voltage or current the desired affect can be altered by a change in resistance.

Semiconductor device physics act differently than basic electronics but the idea of a change in resistance remains the same under non-ideal operating conditions. Electron mobility is how quickly an electron can move which directly affects device performance. This is one of the measurements used in semiconductor design and determines semiconductor device capability.

Since a semiconductor is what it says it is made of resistive materials which lean either toward an insulator or conductor, meaning all materials are essentially resistive. Doping and Ion Implantation introduce certain materials which direct the flow of electrons to reach the desired switching operations. Boron as a p-type and Phosphorus as an n-type, among a few examples, can be introduced to create a desired operation. $\mathrm{N}$-type transistors are known for a higher device mobility but in many cases P-type transistors are needed to complement the $\mathrm{N}$-types for many applications including digital logic and other push-pull networks. This requires a worst case scenario in design to compensate for the P-type mobility rating. 
On top of implanting $\mathrm{P}$ and $\mathrm{N}$ type materials for transistor structure thin film resistors are also added into many designs for any given purpose. These resistors are doped material as well but act more like a conventional resistor. The doping or implanting concentration also is a factor in device mobility, more isn't always better in most cases but to compensate for device reliability and consistent accuracy in the specified operating range a desired target value needs to be found for the material concentration. Expanding and contacting of materials over temperature happen at the atomic level thus altering the device characteristics, resistance, and electron mobility. Higher concentrations of materials can lead to less mobility due to the lack of electrons or electron holes and the same is true in reverse so a balance has to be applied in any design case. I will go further into detail later in the report.

The insulating layer which is grown either with a natural silicon oxide, or with an artificial insulating layer which is the case with most other semiconductor materials and in some cases silicon, has an effect on how the device runs. Dielectrics also play a part in some devices for many of them block the flow of DC current. If the insulating layers or dielectrics are not in proper order then current leakage can occur. Recently since digital manufacturers have been producing lithography sizes lower then $65 \mathrm{~nm}$ it has become a major issue. This is because at that size the insulating layers are only a few atoms thick. Current leakage can affect the potential gain for any transistor based device, because theoretically an ideal device has no current entering or leaving the op-amps or other devices in use inside of an IC, but the real world is not ideal. Since an already accepted amount of leakage happens it has to be controlled, this is why the insulating layers or 
dielectrics are so important. When temperature increases so does the current leakage, this is one of many paracitic effects that must be accounted for in design.

When it comes to design and intent for use the purpose of the device must be established. In terms of operating temperature and other device specifications. The consumer operating range is generally from $0 \mathrm{C}$ to $85 \mathrm{C}$, in most cases narrower than that, where say the military standard (that we know of) is usually between $-55 \mathrm{C}$ and $125 \mathrm{C}$ and military spec expects the range to be guaranteed for worst case scenarios. The difference in temp for consumer range is $85 \mathrm{C}$ and in many cases lower than that, I am choosing consumer and military since they are the two common operating ranges that have the greatest difference. The narrower spec is easier to work with and design for which is why consumer grade parts are cheaper, where the military grade parts have a range of $180 \mathrm{C}$ and must be guaranteed. That is over double and in some cases triple the range that a part is expected to perform to its guaranteed specification, I haven't even touched ranges outside of that yet.

The band gap of all materials used on a device after it is finished has a lot to do with its performance range. Band gap is usually associated more with solid state physics than with electronics but I will give the brief version of it for now. Silicon has a band gap of $1.1 \mathrm{eV}$ (electron volts) where Gallium Arsenide has a gap of $1.4 \mathrm{eV}$. This is the material difference between the valence and conduction band of the material. That $0.3 \mathrm{eV}$ actually makes a big difference, it not only allows GaAs to run at $100 \mathrm{x}$ the frequency with the same amount of heat but GaAs is less sensitive to thermal effects than $\mathrm{Si}$ is. Conductors have a lower band gap where insulators have one that is much higher, semiconductors range from 0.6 to $4.0 \mathrm{eV}$ roughly. A band gap has to exist for a 
switching operation to take place which is what an IC device usually does. As far as which one is ideal there is no one answer but for extreme temperature devices, i.e. drill bit sensors or satellite IC's. A band gap of $1.7 \mathrm{eV}$ or higher is desired for extremely high temperatures or devices that require a very large operating range, there are a few choices to choose from in this range but it is not commonly revealed which ones are used for which application.

Aside from the choice of the material itself, Si actually has a wide range of applications; some manufacturers prefer a more expensive component to insure higher quality or some have a process capable of refining a common material well enough to provide the same results for less cost. There are many factors which can insure a more reliable part that does not require a material change. For some device designs the difference between a consumer part and military part is a matter of material purity, device packaging, environmental isolation, and picking the parts that test closest to the middle of the device specification, a process called binning which I will describe along the way. I can also include other factors like humidify, pressure, and shock into the equation, which I will, but I am starting with temperature since it is the most prevalent in any environment and directly or indirectly affects the other factors.

As stated before where material choice, doping, and the concentration can affect electron mobility this is a best case scenario. Parasitic sub particles and contamination also contribute to the degradation of electron mobility. You can always have a group of chips at test with dead center response but if any forms of contamination or other device flaws are present it can cause problems further down the road. One thing about manufacturing is that it always has to be done in hopes of making a profit. Every 
manufacturer wants to make the best parts on the market with zero impurities and have peak efficiency, some get close to that consistently. However the average consumer is always on a budget where the military is part of the government and money is less of an object for them and they have more of it (Politics aside). In the case of manufacturing, to guarantee that parts meet much tougher requirements the cost goes up.

In most automotive, military, or extreme industrial applications a devices reliability can be the difference between a satisfied customer or a major failure that has safety risks. This is why some customers demand zero defects and perfect reliability over a very wide operating range. What makes high standards so expensive is that there are always defects and chips that do not perform to said standards. From sawing the ingot to final chip packaging maybe one in every ten devices at every processing point meet the requirements. On top of that more qualified individuals are required to work on and inspect each processing point further driving the cost up where before some of those points did not require as much attention.

Packaging of the device can also play a major factor in device reliability and performance. The majority of devices seen in the market have plastic molding around the chip, this is because it is the cheapest form of packaging and the majority of consumer use will never need anything beyond that. For heavy environmental conditions a ceramic package may be required. The advantage to a ceramic package is that it is capable of dissipating heat better and can be hermetically sealed to prevent moisture or anything else from entering and affecting the die. When die age or go under stress the protective nitride layer on top of the die can deteriorate or crack, allowing moisture or air to touch the components of the die. When this happens the oxidation on 
the die will continue to expand again thus altering the resistance and overall device performance. This is one of many reasons to understand what a device is going to be used for or its requirements. A sealed package can keep outside interference out and being ceramic will protect it more and help dissipate heat but ceramic casing is not cheap and the seal also has to be tested which will lead to more chips failing a specific test in line where plastic is cheaper, faster, doesn't require further reliability testing beyond the electrical device test.

Another common packaging method to control device conditions is that seen on computer CPU's to have active or passive cooling through a metal heat dissipater. These methods are usually required on CPU's and other devices that either have a large amount of transistors, a very fast switching operation, or devices that run a high amount of current through them. 


\section{CHAPTER 6}

\section{DIGITAL TECHNOLOGY}

Digital technology is the process if calculating discrete numbers in the form of voltage levels or in any other form of a discrete number. The technology and the idea has been around for many years, Morse code is an example, but became very popular in the 1980's and especially the 1990's. It was around that time frame where discrete voltage levels in the form of a binary 1 or 0 were able to be calculated fast enough on a semiconductor device to produce results to rival and in some cases surpass previously used analog technology.

The factors involved in the emergence of digital technology were cost, reliability, and repeatability. Cost is always a factor in any form of technology. When the transistor was first invented they were very expensive. Factor in all the transistors required to make the logic gates and all the logic gates required to do simple addition and subtraction the cost built up very fast. This is why digital technology did not take off right away.

Repeatability was one of the primary reasons why digital technology was desired for multiple applications. Over time the condition of any device will degrade, especially if used enough. Since analog technology was based on precision values degradation was a major issue. When used in a wireless application any noise from point to point can always cause degradation, which there always is when transmitting over the air. The most common form of storage for analog at the time was magnetic tapes in one form or another. When used enough either being read, written, or re-written the tape would wear or the metallic substance would fade causing degradation in the data stored. 
If you can remember large stereo amps with a large amount of capacitors and inductors, over the air TV transmissions prior to 2009, and VHS tapes you can remember how degraded the video and audio quality was from either being used over time or received over a long distance. This is where digital technology offers a solution.

Digital signal processing is the modern solution for the old analog filters. Where signal filters used to be a combination of various components, now lines of code can be written to filter undesired noise out of a received signal. This is first done by sampling an analog signal and converting it to discrete levels and from there process through a digital filter, basically a program meant to simulate the effects of the components previously used. Since discrete components can wear and decay over time causing undesirable effects in the precision values required digital filters are a solution for this problem requiring CPU power using discrete numbers eliminating the need for precision values.

Reliability was a major factor in the need for digital technology. Since error and decay as previously mentioned was a major issue with analog technology digital offered a solution to these issues. Since digital uses numbers represented in binary, mathematic calculations and computations can be used to compensate for error. Since the voltage levels required representing either a 1 or 0 do not need to be precise and grant room for error decay and slip in precision levels do not factor in to digital technology as much.

A digital signal is really an analog signal shaped to switch from a specified high or low voltage level instantaneously. The switching speed was a major factor on why digital technology took decades to become mainstream. Using TTL (Transistor- 
Transistor) Logic as an example a high voltage level representing a 1 can be anywhere from 2.2 to 5 Volts over a 5 Volt Supply and a low level can be anywhere from 0/GND to $0.8 \mathrm{~V}$, this is how the precision value problem was solved.

As digital has replaced analog technology in many forms the technology still presents multiple issues that must be accounted for in any design. As mentioned before previous digital technologies used 5 Volts as a supply but that has decreased over time, now we are using supplies ranging in the 0.9 to 1.2 Volt range. The clock cycles, switching speeds, and transistor count are the top contributors to power consumption. Power consumption plays a major role in device reliability from reasons to structure integrity to device performance.

When the Intel 8086 was first released in 1978 this was considered a major breakthrough chip as it was the first chip to use the $\times 86$ architecture which is still used today. At the time it was considered top of the line, this chip used a supply voltage of 5 Volts, had a transistor count of 29,000 , had a lithography size of $3 \mathrm{um}$, and had a clock cycle of $5 \mathrm{MHz}$. Fast forward to today and lets compare to Intel's core i7-3770, I am using the Ivy Bridge core i7 made in 2012 because the design is now final where the Haswell/Broadwell is still not finished in its design process. The core i7-3770 has a supply voltage of $1.1 \mathrm{~V}$, transistor count of 1.4 billion, lithography size of $22 \mathrm{~nm}$, and a clock cycle of $3.4 \mathrm{GHz}$.

Why is this important when it comes to device reliability? Thermal design power (TDP) is a major factor that can contribute to the lifespan of a CPU and contribute to its performance or lack thereof. With the previously mentioned CPU's in mind, power consumption jumped from 1.5-2 Watts used in the 8086 to $77 \mathrm{~W}$ used in the $i 7$. What 
makes the TDP even more important is that the $77 \mathrm{~W}$ required is run through a die that is $160 \mathrm{~mm}^{\wedge} 2$, which is roughly about $1 / 4$ the size of a postage stamp.

When lithography sizes are reduced more transistors were able to be packed in the same space as before. Another advantage of this is that the transistors required less power because with shorter distances from point to point (typically drain to source, etc) less current was required to move a charge. This is excellent for saving power but smaller sizes usually mean the feature is less robust, this is where design comes into play.

The load-strength tradeoff is key to device reliability and device lifespan, I am using design techniques for a CPU because it is the one component of a computer that is the most volatile and requires the most attention (Only High end GPU's can be a possible exception).

With smaller features comes the potential for more to be placed in the same area, however smaller features are not capable of as much load as before (using the same materials and same design concept), this is why in design supply voltage and current usage must be accounted for. Even though less power is required for a smaller feature, if more are placed together in the same space previously used heat can build up faster with more activity. Since the heat buildup from the TDP affects the entire chip certain trade offs must be recognized.

Clock speed is also a major factor behind TDP, it is the fastest part of the CPU and sets the intervals of switching for the rest of the CPU. The higher the switching speed the more overall friction is created within the CPU therefore increasing the load and overall TDP. Some methods of curbing this was to decrease the supply voltage, 
with the feature sizes decreasing lowering the supply voltage was not a major issue but did require some compensation via design. Finding an optimal clock speed is also a way to increase the lifespan by decreasing the load and TDP. In most portable devices under clocking and under volting the CPU is a popular idea because there is little room for heat dissipation and in some cases none. Examples of these ideas would be laptops and smartphones.

About 10 years ago clock speeds kept jumping and power usage was going up along with it. The feature size and supply voltage did help curb power consumption and device reliability but around $2006 \mathrm{CPU}$ power consumption was getting near the 100W mark for the desktop PC. The solution to this issue was multi-core CPU applications. What multi-core does is it has multiple Logical CPU's on the same die that share a common clock and can divide the work load between them. The issue is that the software needs to be written or re-written to make use of the new architecture, this is the point when 64-bit operating systems and programs came into play.

Even though technology is obsolete in a matter of a few years, or even a few months, the solution among the consumer population is to replace their CPU or the entire computer should the CPU fail or if it does not meet their specification needs. However replacing hardware does not fit the need of everyone, especially governments and private industry. For some specific applications a specific set of hardware must be used, this condition usually applies to applications that require absolute reliability and consistency. Since a specific application must go through rigorous testing to be qualified to fit a specific need replacing hardware may not be an option, in these cases when a component fails that same specific component must be replaced, including the CPU. 
Since CPU manufacturers only produce a CPU for a specific period of time customers usually ask for a stock pile of parts and some guarantee that the part will last for a specified duration, this is why device reliability and device lifetimes are so important. 


\section{BIBLIOGRAPHY}

Web

Panasonic Industrial, Panasonic Corporation, http://na.industrial.panasonic.com/

TDK Industrial, TDK Corporation, http://www.global.tdk.com

Wikimedia commons, http://www.wikipedia.org

All About Circuits, http://www.allaboutcircuits.com

Basic Electronics Tutorials, http://www.electronics-tutorials.ws

Textbooks

Patrick J O'Conner, Practical Reliability Engineering $5^{\text {th }}$ ed., John Wiley and Sons, Inc., New York City, NY, 2012

Neil Weste and David Harris, CMOS VLSI Design $4^{\text {th }}$ ed., Addison-Wesley, Boston, MA, 2011

John G. Proakis and Dimitris K. Manolakis, Digital Signal Processing $4^{\text {th }}$ ed., Prentice Hall, Upper Saddle River, NJ, 2006

Behzad Razavi, Design of Analog CMOS Integrated Circuits, The McGraw-Hill Companies, Inc., New York City, NY, 2001

David M. Pozar, Microwave Engineering $4^{\text {th }}$ ed., John Wiley and Sons, Inc., New York City, NY, 2012 